\title{
IFLA SPECIAL INTEREST GROUP (SIG) ON LIBRARY \\ PUBLISHING. 2019 MIDTERM MEETING \\ (DUBLIN, 28. FEBRUAR-1. MÄRZ 2019)
}

\author{
von Elisabeth Stadler und Markus Stumpf
}

Zusammenfassung: Von 28. Februar bis 1. März 2019 fand in Dublin/IRL eine von der International Federation of Library Associations and Institutions veranstaltete Konferenz zum Thema „Library Publishing" statt. Vortragende aus Europa und Nordamerika sprachen an der Dublin Business School (DBS) zu Publikationsprojekten, Herausforderungen und Perspektiven von Bibliotheken als Verleger und Herausgeber von Publikationen.

Schlagwörter: Universitätsverlag; Bibliotheksverlag; Publikationsdienst; Tagungsbericht

\section{IFLA SPECIAL INTEREST GROUP (SIG) ON LIBRARY PUBLISHING. 2019 MIDTERM MEETING (DUBLIN, FEBRUARY 28-MARCH 1, 2019)}

Abstract: On the $28^{\text {th }}$ of February and $1^{\text {th }}$ of March the International Federation of Library Associations and Institutions organized a conference about "Library Publishing" in Dublin, Ireland. Hosted by Dublin Business School (DBS) lecturers from Europe and North America discussed publication projects, challenges and perspectives of library publishing.

Keywords: University Press; Library Press; Library Publishing; Conference report

DOI: https://doi.org/10.31263/voebm.v72i2.3029

(c) Elisabeth Stadler, Markus Stumpf

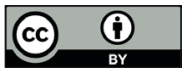

Dieses Werk ist lizenziert unter einer

Creative-Commons-Lizenz Namensnennung 4.0 International 
Am 28. Februar und 1. März 2019 fand in Dublin/IRL eine von der International Federation of Library Associations and Institutions (IFLA) veranstaltete Konferenz zum Thema „Library Publishing“ statt. 21 Vortragende aus Bibliotheken bzw. Bibliotheksverlagen in Europa und Nordamerika sprachen an der Dublin Business School (DBS) zu Publikationsprojekten, allgemeinen und technischen Herausforderungen und Software-Entwicklungen ihrer Institutionen. Eine Panel-Diskussion rundete die Veranstaltung ab und verschaffte nochmals einen Überblick über die neuen Perspektiven, aber auch Herausforderungen und Hürden, mit welchen sich Bibliotheksverlage konfrontiert sehen.

Nach einleitenden Worten von Ann Okerson (International Coalition of Library Consortia), Philip Cohen (Präsident der Library Association of Ireland) und Jane Buggle (DBS) widmete sich der erste Tagungsblock Fallstudien für das Publizieren in Bibliotheksverlagen und deren Herangehensweisen. Suzanne Cady Stapleton (University of Florida) stellte in ihrem Statement die Florida Online Journals vor. Diese sind seit 2000 online Open Access verfügbar, seit 2002 wird auch eine Volltextsuche unterstützt. Sie sieht es als eine ihrer Hauptaufgaben an, Wissenschaftlerlnnen bestmöglich über die Anforderungen wissenschaftlichen Publizierens zu informieren (ORCID, Metadaten, ethische Grundlagen, ...). Christina Lenz (Stockholm University Press) etablierte 2013 den Verlag als reinen Open Access-Verlag und sieht Open Access und Open Science als ihre „Mission“. Layout und Herstellung werden nicht mit angeboten, der Schwerpunkt liegt in der Unterstützung der Autorlnnen und HerausgeberInnen v. a. auch hinsichtlich rechtlicher Fragen im Bereich der unterschiedlichen Lizenzen. Auch die White Rose University Press präsentiert sich als reiner Open Access-Verlag, wie Tom Grady betont. Die Publikationen können in den diversen Formaten heruntergeladen, aber auch als Hardcopy bestellt werden. Der Verlag konnte 2018 drei Bücher auf den Markt bringen und betreut vier Online-Journals. Den Abschluss des ersten Tagungsblocks bildete das Statement von Ally Laird, die als ehemalige Mitarbeiterin bei SpringerNature ihre Erfahrungen bezüglich der Arbeit mit Autorlnnen und den sich daraus ergebenden Herausforderungen in ihre Arbeit beim Verlag der Penn State University Libraries einfließen lassen kann.

Das zweite Panel am Donnerstagvormittag war den Schlagworten Ausbildung und Mentoring gewidmet. Melanie Schlosser vom Educopia Institut stellte das „Library Publishing Curriculum" vor, das aus einer Zusammenarbeit von Educopia Institute, Library Publishing Coalition (LPC), Public Knowledge Project (PKP), NASIG und BlueSky to BluePrint hervorgegangen ist. Dieses Fortbildungstool für Bibliothekarlnnen ist aufgrund 
der CC-BY-Lizenz kostenlos und offen nutzbar (s. https://educopia.org/ library-publishing-curriculum/). Michelle Wilson (Columbia University, Digital Publishing Librarian) referierte über die Herausforderungen, die sich beim Publizieren von Journals ergeben, da a) selten ein Back-Office für den Verwaltungsaufwand vorhanden ist, b) Grundwissen des Publizierens meist fehlt, $c$ ) es einen Bedarf an Schulungen zu diversen Tools und Software gibt und d) es oft keinen Zugang zu und Erläuterung von Dokumentationen gibt. Wilson zeigte auf, wie diese Themen mithilfe von Workshops aufgearbeitet werden. Kevin Stranack (Simon Fraser University Library \& Managing Director) berichtete über den Versuch, im Rahmen einer Lehrveranstaltung mit Studierenden alle Aspekte der Entstehung eines Journals durchzuarbeiten. Gearbeitet wurde mit Open Journal Systems (OJS) und die Studierenden agierten als Autorlnnen und Reviewerlnnen, erhielten Informationen zu juristischen Aspekten, befassten sich mit den Stadien der Überarbeitung etc. Journal-Manager bzw. Layouter war der Bibliotheksverlag, der Vortragende war der Herausgeber. Das Projekt wurde sowohl von den Studierenden als auch von den Vortragenden und der Bibliothek sehr positiv bewertet, allerdings muss auf den großen Arbeitsaufwand hingewiesen werden. Im persönlichen Gespräch wurde mitgeteilt, dass die studentischen Arbeiten aus Qualitätsgründen nicht ins Repositorium eingespielt werden würden. Helge Høivik (Universitätsbibliothek der OsloMet) beendete die Vormittags-Session mit einem Vortrag über MOOCs und digitale Lehrbücher an der OsloMet Universität.

Am Nachmittag standen dann Geschäftsmodelle und Nachhaltigkeit auf dem Programm. Trude Eikebrokk (Universitätsbibliothek der OsloMet) eröffnete mit einer Präsentation, die sich mit der Verwendung von Open Journal Systems (OJS) für Publikationen an der Universitätsbibliothek der OsloMet beschäftigte. Neben dem ursprünglich geplanten technischen Support ist ihr Team mittlerweile Anlaufstelle für alle Fragen im Publikationsablauf bis hin zum Review der Beiträge. Daher wird mittlerweile ein umfassendes Service angeboten, das auch Trainings für Herausgeberlnnen beinhaltet. Daraus entwickelte sich ein Geschäftsmodell, da man diese Dienstleistungen um ca. EUR 7.200.- pro Jahr auch für externe Journals öffnete. Alex Kouker (Dublin City University) und Jack Hyland (Manager bei IRel) referierten über nachhaltige Geschäftsmodelle für neue Universitätsverlage. Die von ihnen vorgestellte Initiative nennt sich Open Access Exchange, Irland (OAeX, Irl) und weist Ähnlichkeiten zu den Modellen von Open Library of Humanities (OLH), Knowledge Unlatched und anderen Initiativen auf. Mit OAeX soll den Bibliotheken die Freiheit gegeben werden, selbst zu entscheiden, welche OA-Publikationen sie unterstützen, wo- 
bei nicht nur Journals im Fokus stehen, sondern auch Monografien oder auch die Druckvorstufe etc. Das Modell sieht vor, dass die Bibliotheken die Beiträge finanzieren und OAeX die Verteilung der Mittel verwaltet. Momentan ist dies noch eine irische Initiative, Ziel ist es aber, international zu agieren. Rachel Lammey (CrossRef) stellte in ihrem Beitrag die Arbeitsweise von CrossRef vor und unterstrich dabei, dass die Vergabe eines DOI nur ein kleiner Teil der Aufgaben ist. Angeboten wird hier ein breites Spektrum an Dienstleistungen, die sicherstellen sollen, dass Forschungsdaten gespeichert und verlinkt werden. Wobei zu den verarbeiteten Metadaten nicht nur die bibliografischen Angaben, Stichworte, Abstracts etc. zählen, sondern z. B. auch Informationen bezüglich der verwendeten Lizenz wichtig sind. Die Verwendung von CrossRef ist jedoch nicht nur auf Artikel in Journals beschränkt, sondern es können auch Monografien, einzelne Kapitel aus Büchern, Konferenzberichte, Dissertationen, Reviews etc. registriert werden.

Das letzte Panel des ersten Konferenztages beschäftigte sich mit Auswirkungen auf die Gesellschaft. Hellen Fallon (Universität Maynooth) präsentierte die Entstehungsgeschichte der Publikation der Korrespondenz mit Ken Saro-Wiwa (1941-1995). In diesem einzigartigen Archivmaterial befindet sich die private Korrespondenz von Ken Saro-Wiwa mit der irischen Nonne, Schwester Majella McCarron, die jahrelang das Ogoni-Volk im Kampf gegen die Ölverschmutzung in Nigeria begleitete. Die Briefe und Gedichte aus der Zeit vor seiner Ermordung in der Haftanstalt wurden in Kooperation mit seiner Familie herausgegeben und unterstützen nun weltweit den Kampf der Ogoni für eine intakte Umwelt. Gerard Connolly und Niamh Walker-Headon, beide Bibliothekare an der Technischen Universität Tallaght Campus, berichteten von einem Publikationsprojekt eines Prosa-Wettbewerbs (IT Tallaght Short Story Competition), mit dem Selbstveröffentlichungswerkzeug Kindle Direkt Publishing (KDP) von Amazon. Während die Herstellung und Bewerbung der Bücher relativ einfach ist, sind ihre Erfahrungen mit der Rezeption jedoch nicht so zufriedenstellend. Mittlerweile ist allerdings bereits der dritte Band erschienen. Jane Buggle (DBS) führte in ihrem Bericht über die irische Minderheit der Irish Travellers ein und über die Möglichkeiten, dieser marginalisierten Bevölkerungsgruppe Irlands mit Bibliothekarlnnen als Herausgeberlnnen eine Stimme zu geben. Konkret wurde dies anhand des Sonderhefts über Minoritäten und Indigene Bevölkerung des Studies in Arts and Humanities Journal (SAHJ) dargestellt, das anlässlich der offiziellen Anerkennung der Irish Travellers als ethnische Minderheit durch die irische Regierung im Jahr 2017 in der DBS Library Press entstanden war. 
Der zweite Tag widmete sich zunächst der internationalen Zusammenarbeit. Isabella Meinecke (Hamburg University Press der Staats- und Universitätsbibliothek Hamburg) präsentierte die Arbeit der Association of European University Presses (AEUP). Das Netzwerk der Universitätsverlage besteht seit 2010 und umfasst mittlerweile 39 Mitglieder aus 18 Ländern. Ein derzeitiger Schwerpunkt ist die Etablierung von Standards für elektronisches Publizieren. Promita Chatterji (Product Manager for Digital Commons) und Aajay Murphy (Repository und Publishing Manager an der Kennesaw State University) demonstrierten anhand ihrer Fallbeispiele, wie sie internationale Partnerschaften und Verbindungen von Bibliotheken für ihre Projekte nutzen. Sie haben sich den freien Zugang zu den Ressourcen zum erklärten Ziel gesetzt. Murphy z. B. managt über 20 Journals mit unterschiedlichem Betreuungsbedarf, die alle ihre Inhalte Open Access zur Verfügung stellen. Autorlnnen bzw. Reviewerlnnen sind dabei über den gesamten Globus verstreut. Auch Chatterji betonte die Wichtigkeit des „Global Impact“, um nicht nur die Sichtbarkeit zu erhöhen, sondern auch eine möglichst breite Streuung unter den Autorlnnen bzw. HerausgeberInnen und Reviewerlnnen zu erreichen. In der Diskussion kritisch angemerkt wurde die Partnerschaft von Digital Commons zu Elsevier. Ursula Arning (ZB Med) berichtete über das Open-Access-Publikationsportal für Lebenswissenschaften PUBLISSO. Hier können alle Wissenschaftlerinnen und Wissenschaftler aus den Bereichen Medizin, Gesundheitswesen, Ernährungs-, Umwelt- und Agrarwissenschaften ihre Forschungsarbeiten und -daten veröffentlichen und sich über Open Access und Publikationsmöglichkeiten in ihren Bereichen beraten lassen. Christopher Hollisters (University of Buffalo) Vortrag hatte als Fallbeispiel die Zeitschrift Communications in Information Literacy (CIL) zum Inhalt, deren Mitbegründer und Mitherausgeber er ist. Dieses unabhängige Wissenschaftsjournal wird von der Bibliothek herausgegeben und widmet sich inhaltlich dem Bereich der Informationskompetenz. Seit der Gründung 2007 sind 13 Hefte erschienen, alle Open Access verfügbar. Christopher Hollister hat es sich zum Ziel gesetzt, "Scholarly Communication“ in ein offenes System zu transferieren, was er derzeit als deren interimistischer Leiter an der Universität von Buffalo intensiv verfolgt.

In der Abschlussdiskussion mit Ann Okerson, Lars Egeland, Melanie Schlosser, Niamh Brennan, Jane Buggle und Elisabeth Stadler (Grazer Universitätsverlag) wurde die Schlüsselrolle von Bibliotheken im derzeitigen Wandel des Publikations- und Verlagswesen diskutiert. Als Fazit konnte festgehalten werden, dass das Bibliothekswesen komplett neu gestaltet wird - es findet quasi ein reengineering- und rebranding- sowie ein digi- 
tal-curation-Prozess statt, der neue Fähigkeiten und Fertigkeiten von zukünftigen Bibliothekarlnnen verlangt. Die Univeritätsbibliotheken als Verlage sollten sich dabei jedenfalls mehr als Partnerinnen denn Dienstleisterinnen ihrer Universitäten positionieren.

Eine Tagungsdokumentation mit den Vortragsfolien und Videos ist unter https://www.ifla.org/node/92080 abrufbar. An einer späteren Publikation der Ergebnisse wird gearbeitet.

\author{
Mag. ${ }^{a}$ Elisabeth Stadler \\ Universität Graz, Grazer Universitätsverlag \\ E-Mail: elisabeth.stadler@uni-graz.at
}

Mag. Markus Stumpf, MSc

ORCID: http://orcid.org/0000-0003-4946-9988

Universität Wien, Bibliotheks- und Archivwesen

E-Mail: markus.stumpf@univie.ac.at 